\title{
Together or Not? Exploring Stakeholders in Public and Permissionless Blockchains
}

\author{
Roman Schmid \\ University of Zurich \\ roman.schmid@bluemail.ch
}

\author{
Rafael Ziolkowski \\ University of Zurich \\ rafael.ziolkowski@gmail.com
}

\author{
Gerhard Schwabe \\ University of Zurich \\ schwabe@ifi.uzh.ch
}

\begin{abstract}
The emergence of blockchain projects enables new ways of collaboration between untrusted parties. Each of these projects, however, only exists because stakeholders of these projects find common ground. If this common ground is not found, blockchains are forked - organizationally and technically - which endangered major blockchain systems like Bitcoin or Ethereum. To assure the operation of such projects and, thus, to improve their governance, it is crucial to understand their stakeholders. This research conducted a literature review and a survey to (1) identify blockchain stakeholders and to (2) understand their interests as well as underlying motives for their interests. This research has two main contributions: a stakeholder map, which serves as a lens to study stakeholders of public blockchains, and exemplary insights from the application of this lens comprising of 74 survey responses. Consequently, this research provides a novel tool for stakeholder analysis in academia and practice to improve blockchain governance.
\end{abstract}

\section{Introduction}

Within the past few years, public blockchain projects such as cryptocurrencies have managed to change our perception of prevailing economic and organizational infrastructures. They allow radical new ways of collaboration between untrusted parties, largely bypassing centralized intermediaries. Above all, Bitcoin (BTC) represents one of the first effectively realized attempts to eliminate the central party in a transactionbased financial system. As an implicit response to the global financial crisis of 2007-2008, BTC seeks to supersede the need for trust in such central fiduciary institutions by operating as a so-called trust-less technology [1]. Ultimately, the BTC project rendered one premise: "[...] to solve issues of social coordination and economic exchange by relying, only and exclusively, on technical means" [2, p. 1]. This shall be enabled by shifting the controlling instance, which oversees transactions between untrusted parties, away from centralized institutions (such as banks) to a technology-based solution (in this case, a blockchain enabled distributed-ledger system). By concept, this leads to a general independence of centrally governed infrastructures, as stakeholders in such peer-to-peer (P2P) networks rely mostly on their inherent technological regulations.

A closer look at the allegedly distributed, technocratic governance of established cryptocurrencies reveals, however, that expectations regarding decentralization do not necessarily coincide with reality. These projects often have an underlying autocraticmechanistic power structure $[2,3]$. Demagogic public appearances, exertion of influence based on technical expertise, and publicly held disputes about the future development of such projects allow certain key figures to emerge as central so-called benevolent dictators [4, 5]. The formation of interest groups, where contributed resources are consolidated (such as hash-power in mining pools), reveals another problematic distribution of centralized power in systems, whose primary intention is to use a decentrally-governed infrastructure [6]. Lastly, large cryptocurrencies experienced various harsh conflicts, which endangered their existence; often these conflicts led to splits, so-called forks, within these projects [2].

The root of these problems lies in the formal independence of stakeholders in blockchain systems. Public blockchain projects consist of several, formally independent participants, for example developers and validators, to develop, maintain, and run their systems. At the same time, every stakeholder might have different interests around a blockchain system, with the consequence of divergent expectations about how such a system should be built or run. This is particularly important as, when this common ground among stakeholders is missing, blockchain projects fork both organizationally (split of communities underpinned to each blockchain project) and technically (creation of a new, incompatible ledger). To illustrate these interests, also considering recent blockchain developments [7], 
one might ask, what a blockchain stakeholder primarily cares about: a token's price or technical evolution? Do individuals mind technological decisions? Are they involved in decision-making? Do they even want to be involved in decision-making? To safeguard and align incentives and interests of different stakeholders plays a key factor for such decentralized projects to persist [8, $9,10,11]$, which is at the heart of the research stream of blockchain governance.

Against the background shown above, this research, in line with several other researchers $[12,13]$, argues that blockchain stakeholders are not sufficiently understood in current research on blockchain governance. A grounded understanding of blockchain stakeholders is, at the same time, a prerequisite for blockchain systems to prevail as it allows to develop or adjust a blockchain's governance structures. As of now, there are several approaches in literature that try to capture these complex governance structures among stakeholders in public blockchains $[2,9,10,11]$. To improve the understanding of stakeholders of public blockchains and, consequently, to improve their governance, this research shows first a classification of such stakeholders and then shows their interests, their motivations behind their interests, and the design features of blockchains they were particularly interested in. Consequently, our research was guided by the following research questions:

RQ1: What are the stakeholders of public and permissionless blockchain projects?

RQ2: What are the interests of these stakeholders?

To answer our research questions, this research reports from the findings of a literature review and a qualitative and quantitative analysis of survey results comprising answers of 74 stakeholders of various public and permissionless blockchains.

This paper is structured as follows: section two introduces related work on stakeholders in the wider frame of blockchain governance. Section three then shows our methodological approach regarding framework development and operationalization, while section four shows the results of the latter. Within section five, we contextualize our empirical findings against the background of blockchain governance and conclude this research in section six.

\section{Related Work}

To introduce relevant concepts to our research, this section first introduces views on blockchain governance, and, subsequently, highlights the importance of a stakeholder-centric view.

\subsection{Views on Blockchain Governance}

There are several approaches in academia that try to capture the phenomenon of decentralized governance in public blockchain projects. For example, De Filippi and Loveluck [2] describe the emerging conflicts within communities of public blockchains. Thereby, they introduce the distinction between governance by infrastructure and governance of the infrastructure. Essentially, they explain to which extent consensus can be found and maintained by strict technological means, i.e., implemented consensus protocols. On the same line of thought, Lumineau, Wang, and Schilke [11] examine the differences between the governance of blockchain and traditional contractual and relational governance. They conduct an analysis of blockchain governance to further assess its feasibility as a new way of organizing collaborations. Against the background of blockchain governance, they relate the term cooperation to the alignment of interests, whereas aligning expectations is associated with coordination [11]. Regarding the already mentioned dynamics of interdependent stakeholders in public blockchains, they emphasize this research's area of interest by concluding: "We see particular interest in analyzing the social construction of blockchain governance and in studying how different stakeholders shape their design and meaning across specific contexts as a function of their resources, powers, or capabilities" [11, p. 17].

To capture the power dynamics in public blockchains Beck, Müller-Bloch, and King [9] extend the IT governance framework developed by Weill [8], which consists of the three dimensions decision rights, accountability, and incentives. Based on this framework, they express the importance of aligned incentives in such blockchain projects, and their corresponding analysis. They outline the consequences of a failed consensus finding in this regard as follows: "Incentives are absolutely crucial for the blockchain economy to function effectively, because incentives are necessary to achieve consensus that forms the backbone of the blockchain. Unless incentives are properly aligned, the nodes [i.e., stakeholders] of the blockchain will not contribute to consensus. Improper incentive alignment could threaten the integrity of the entire blockchain make the blockchain economy untenable" [9, p. 1029].

Schmeiss, Hoelzle, and Tech [10] tackle the governance aspect of public blockchains based on the paradox of openness. As such blockchain projects can be seen as platform ecosystems, they apply the overarching dimensions access, control, and incentives in their framework to elaborate the issue of how such projects enable maximum openness for mutual 
innovation while ensuring value capturing for all involved stakeholders.

Lastly, Musiani, Mallard, and Méadel [14] describe different facets of user profiles in public blockchains and their associated role of shifting such projects towards more de- or centralization. Eventually, they point out the importance of understanding how different stakeholders situate themselves in and behave around blockchain projects.

\subsection{The Importance of Stakeholders in Blockchain Governance}

This research builds on the abovementioned insights and argues that the different views on blockchain governance have a common denominator: interest alignment among stakeholders plays a crucial role in public blockchains. This work therefore aims to thoroughly identify existing stakeholders and their interests with a higher level of detail than we found in present literature, to obtain a sound basis to further assess the dynamics within blockchain governance.

Indeed, the aspect of decentralization in public blockchains has two different components: a technical and an organizational one. We believe that the examination of organizational challenges within blockchain communities is not yet concluded, and that a suitable model that captures the interest of involved stakeholders is needed. Several researchers describe the application possibilities, and thus implied stakeholder's needs and expectations of blockchain projects [15]. Wingreen, Kavanagh, John Ennis, and Miscione [16] aim to capture the value systems behind cryptocurrencies and subsequently categorize stakeholders in different groups, based on shared values. Their categorization, however, does not account for overlapping stakeholder types, e.g., stakeholders belonging to two of their categories.

The study of these proposals left the impression of properly set approaches, but with a too low granularity in used frameworks to adequately mirror the individual interdependencies among different stakeholders. An often-observed generalization of stakeholder groups, in our perspective, distorts the view on stakeholder dynamics within public blockchains. Particular interests must be captured as such, as virtually every participant, i.e., contributing node, decides unilaterally whether to participate in a public blockchain project or to withdraw committed resources. As described with the paradox of openness [10], these particular interests are mirrored in the individual value capturing for every partaker in such a platform economy. Once more, public blockchains rely on the safeguarding and alignment of these (deviating) interests of different stakeholders to persist.

\section{Methodology}

In this research, we conducted five steps consisting of an (1) literature review, (2) empirical data collection, (3) stakeholder map development, (4) survey design and conduct, and (5) data analysis. Figure 1. below gives an overview of these five activities, which we detail in the following one by one. The research was carried out by a group of three, with one researcher taking the lead, assisted by two senior researchers in recurring review and feedback cycles.

\section{Framework Development}

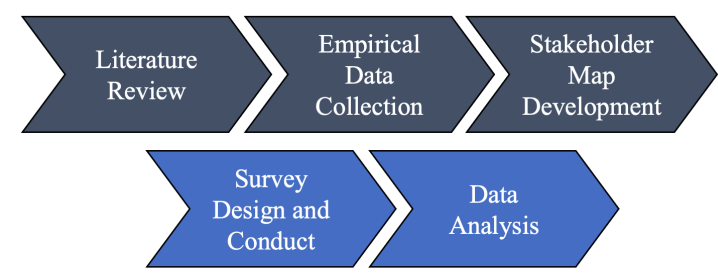

Framework Operationalization

Figure 1. Methodology structure

Literature Review. To eventually conduct a survey on blockchain stakeholder interests, at first, we strove to understand the current state of a stakeholder-centric research within the research stream of blockchain governance. Consequently, we created a preliminary overview of existing frameworks that addresses blockchain stakeholder interests by the means of a literature review. Amongst others, we used search terms like (public and permissionless) blockchain projects, governance discourse, stakeholders, interests, incentive schemes and combinations of these to find and narrow down relevant literature on academic search engines (mainly swisscovery.slsp.ch and scholar.google.com). We also utilized already analyzed major incidents like the block-size dispute of BTC in literature to reveal deviating interests of stakeholders in public blockchains. Eventually, our preliminary framework relied on the final set of 30 academic papers.

Empirical Data Collection. To answer our research questions, we aimed to develop a model (stakeholder map) that shows existing stakeholders (RQ1) and their interests and motives (RQ2) in public and permissionless blockchain projects. We found that the academic literature cannot move with the fast pace with which new projects and concepts appear in the field of blockchain technology. In this regard, we collected in total 32 articles (grey and non-academic literature) on dedicated online platforms (mainly medium.com, cointelegraph.com, and coindesk.com) to understand the actual pulse of blockchain communities in practice. This qualitative data, combined with the insights from 
the preliminary literature review, formed the basis for our stakeholder map development.

Stakeholder Map Development. During an iterative coding process, we analyzed our gathered articles with an appropriate qualitative data analysis software tool called MAXQDA. To build our codebook, we used academic literature as a reference for an initial framework (top-down). This framework, then, was enriched with empirical insights (bottom-up) from our empirical data collection. We isolated single aspects, such as a single interest, motive, or stakeholder, which we eventually embedded and collated in our stakeholder map. Following common principles of qualitative data analysis, we designed our codebook with great care to account for clearly defined, and thus comparable facets on the same level of detail. In total, we applied several dozens of codes and subcodes to over 30 articles and marked over 840 different segments with one or multiple codes, defining and delineating all single aspects of our stakeholder map. After its development, which involved several researchers, we back-tested the developed model by conducting another, complementary literature review. Eventually, using further permutations and alterations of our key search queries on different academic databases, we were able to omit and collate several of our codes. We did so to meet the issue of different sources describing the same circumstance with distinct, but conceptually related keywords. This formed a first validation of our model.

Survey Design and Conduct. Our developed stakeholder map, which we derived from literature (academic, grey, and non-academic), formed the first part of our results, and provides us with a preliminary answer to our research questions. As a next step, we decided to operationalize our model by the means of a survey and gather quantifiable data from communities of current public blockchains. This approach would ultimately form a second validation of our model, as we aimed to assess the occurrence and importance perception of identified aspects in our stakeholder map. We designed the survey to consist of five parts: (1) actors (choosing one or multiple roles), (2) drivers behind their motivations, (3) design features desired from a blockchain system, (4) concluding questions (e.g., how conflicts in one's project were dealt with), and (5) demographics. The survey covered both quantitative questions by use of a Likert scale and freetext answers, which were then qualitatively analyzed. The survey participants were able to indicate multiple aspects which drives them in public blockchains; their proportional occurrence is displayed in Table 1. Driver distribution in section 4.2 below. To assess the importance of interests (Design Features), the respondents were asked to rate these aspects using the following linear scale: 1 (not important) $-2-3$ (neutral)
- 4 - 5 (very important) - 0 (I do not know). These averaged, i.e., weighted importance ratings are shown in Table 2. Interest distribution (section 4.2). Due to space constraints, we cannot describe the survey design in detail here; details can be provided upon request.

The survey was published on over 20 distribution channels, which we carefully evaluated to account for active public and permissionless blockchain projects (mainly online discussion forums, e.g., reddit.com, bitcointalk.com, etc.). In these forums, we addressed individuals that are actively involved in public and permissionless blockchain projects (covering all possible actor types in our stakeholder map). Stakeholders of private / consortium blockchains were not considered for this survey as filtered per the first survey question. Within a period of three weeks in March 2021, we collected in total 120 survey responses, of which 74 responses were fully answered. Within this research, we only report from the 74 full responses.

Data Analysis. We utilized the survey for both model validation and exploration, by allowing users to add answers if they felt that our model did not account for their role, driver, interest, or blockchain feature. Based on our survey results, we identified stakeholder clusters and characterized them by allocating drivers and design features of our stakeholder map to these clusters. Thereby, a stakeholder is assigned to such a cluster if both associated actor types are embodied simultaneously. Further, we examined the proportional occurrence (percentage) of drivers, and a linear weighted importance rating scale regarding design features. We followed this approach to examine the root of stakeholder interests by evaluating the driving forces (motives, incentives) that cause individuals to partake in public blockchains in the first place, followed by their respective importance perception regarding the design features of such projects, i.e., how stakeholders expect such projects should be manifested. Supported by quantitative data, this method analyzes the interests of stakeholders in public blockchains (RQ2) in both a coherent and comparable manner.

\section{Results}

\subsection{Stakeholder Map}

To identify the stakeholders of public blockchains (RQ1), and the interest of these stakeholders (RQ2), we synthesized our empirical insights in a stakeholder map (Figure 2.). It comprises three main entities: Blockchain (BC) Governance, Drivers, and BC Design Features, which we will detail in the following. The allocation of these identified aspects to stakeholders (based on our survey results) is covered in the subsequent sections. Furthermore, we take the BC Environment into account, 


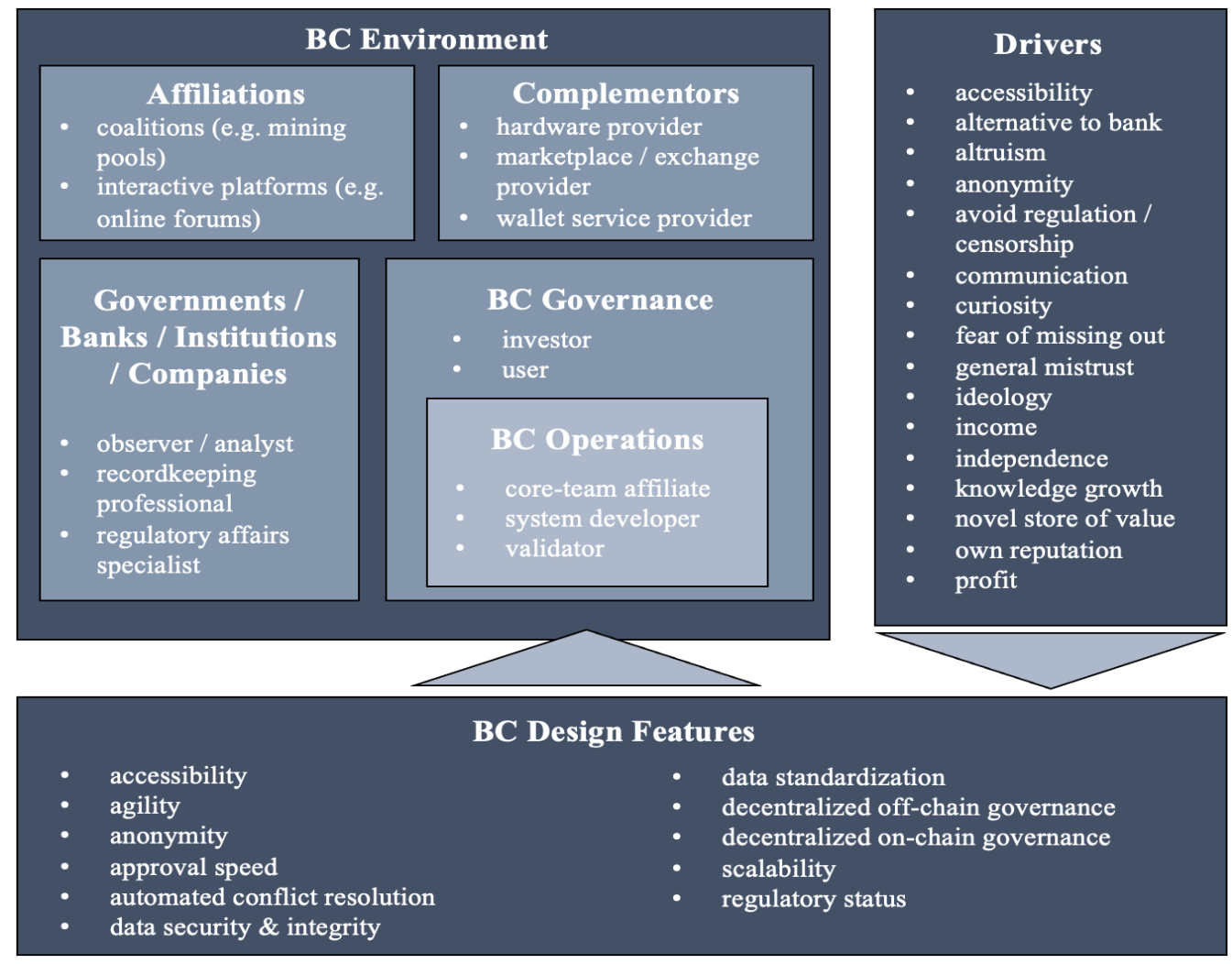

Figure 2. Stakeholder map

of which the $B C$ Governance is part. Please note, that we are only able to show excerpts of the stakeholder map and our utilized sources here due to space limitations. The following descriptions are based on our qualitative data analysis.

Blockchain Governance and Operations. To depict the involved parties of public blockchains, we enclosed its main stakeholders (RQ1) in the $B C$ Governance frame. This entity further includes Operations and is itself embedded in the $B C$ Environment. We found that core-team affiliates, system developers and validators are the bare minimum of required stakeholders for a public blockchain to operate. Through their participation, a first project instance gets initiated, the code on which the technical infrastructure is based is created and maintained, and transactions on the implemented protocol are validated.

Upon operation, the utilization of such projects depends on its users and investors. The latter applies to individuals that are involved by possession and active trading of corresponding crypto assets. Whereas their participation is narrowed to these trades, users do make use of the provided blockchain-inherent functionalities (such as conducting payment transactions).

All these stakeholder types contribute individual resources, and all these stakeholders strive to protect their interests. Financing, technical expertise, a broad user basis to establish network effects; they are all vital aspects for public blockchains. However, these projects only exist if their stakeholders find common ground. In this regard, besides the technical-based consensus protocols, there is no central instance that governs and attends to value distribution among these differently contributing stakeholders. To comprehend this delicate relationship, we use the entity Drivers to capture what are the incentives and motives that drives stakeholders to partake in public blockchains, and $B C$ Design Features (refers to stakeholder interests) that describe realization aspects of these projects, whereby some of these aspects may come with certain trade-offs.

Blockchain Environment. To contextualize the infrastructural setting of public blockchains, we outlined the $B C$ Environment, which contains the $B C$ Governance itself. From established structures, which we detail in the following, there are several emanating forces with the potential to influence or even determine the behavior of stakeholders.

There are collections of stakeholders, that are part of an interest group and often partake in a project with the same activities, i.e., they contribute the same kind of resources. We label these units as coalitions (e.g., coredevelopment teams or mining pools). As they contribute 
shared resources to a shared goal, they are characterized by their leverage power over independently acting stakeholders. We provide an example based on mining pools, as many public blockchains are (still) based on a proof-of-work consensus protocol. In mining pools, the joined computing power of validators leads to higher, steadier, and more predictable mining rewards as if the validators would act on their own. Such collectives of validators pursue several strategies to either increase these rewards or earn them instead of other competitors. These leveraging tactics, however, can form friction points with other stakeholders that are not contributing through validation, e.g., by leading to higher transaction fees, impaired security, or disputable amendments to the implementation (cf. the block-size dispute of BTC) [2, 6]. This potential to undermine initial premises of public blockchains poses a viable threat for lasting consensus finding across all involved stakeholders $[17,18]$.

The organizational aspect of public blockchains is guided by decentralization, and we found the same for the aspect of communication. Interactive platforms, such as online forums, serve as important channels to distribute and broadcast information between different stakeholders. However, there are potential issues when certain platform members take an influential role and exploit their status and arise as so-called benevolent dictators. Newcomers of the blockchain market might inform themselves about their opportunities on such interactive platforms. There is a potential threat regarding scams, as fraudulent stakeholders take advantage of the existing naivety and ignorance of such stakeholders regarding blockchain technology. Some of these individuals might be prone to follow sudden (investment) trends, initiated or incited by demagogical figures on such platforms. Eventually, the sheer mass of such latently steered stakeholders might have a palpable influence on the crypto markets and thus the further development of public blockchains in general.

There are several well-established complementors in the blockchain economy, mainly hardware and software providers, and marketplaces and exchanges. They show a multilayered influence and leverage on stakeholders, which we detail in the following. Taking up the example regarding public blockchains with a proof-of-work architecture, hardware providers profit from increasing technical standards of validation (mining) equipment, due to the high competition among stakeholders that contribute to projects by validating transactions. High-quality (i.e., the most efficient) hardware are rare and expensive, additionally, they are often specialized on a consensus protocol that is proprietary to just one or a few public blockchains. This potentially impairs the agility of validators to switch to different projects if the market changes. Moreover, the increasing complexity of setting up such hardware, combined with long delivery times and rapidly changing markets, sets the market entry barrier very high for new stakeholders who intend to contribute by validating transactions. Newly forming projects might therefore depend on already established networks of dedicated validators, who in turn depend on hardware providers.

Marketplaces and exchanges, as well as wallet service providers provide the aspect of accessibility to the blockchain economy. Amongst others, they manage to attract newcomers by transforming crypto-based assets into convenient investment instruments. For the blockchain economy itself, one can argue this to be a double-edged sword. On the one hand, blockchain projects might struggle to acquire sufficient funding through investors. Eased access to invest (marketplaces and exchanges) and hold (wallet service providers) might help to reach a needed threshold. On the other hand, many of these attracted stakeholders might have little knowledge about their invested public blockchain projects, as they are presumably guided by (quick) profits only.

Drivers. We use Drivers as an umbrella term for motives and incentives that drive stakeholders to participate in public blockchain projects. The aspects of this stakeholder map entity have a broad spectrum: they might originate from intrinsic (e.g., altruism) or extrinsic (fear of missing out) stimuli, are pecuniary (profit) or non-pecuniary (curiosity) based or grasp other existing behavioral forces. The further allocation of these aspects to stakeholders might reveal latent friction points in shared consensus finding, as mutual interests might not be rooted in the same rationale.

BC Design Features. To meet the interests of the stakeholders, public blockchain projects can focus on various design features, such as data security \& integrity or anonymity. There might be trade-offs between some of these aspects, for instance security must be weighed against efficiency. Our identified aspects in public blockchains recurrently form friction points among stakeholders. Initial promises, i.e., often the proprietary identity aspect of such projects, can be mirrored by these design features. Using Drivers to grasp the impulsions of stakeholders, the entity of $B C$ Design Features serves as a common denominator across the entire spectrum of actors. Individuals who are different regarding their contribution towards a public blockchain (e.g., financial, know-how) can nonetheless gather around a key design feature that is equally important to all.

\subsection{Stakeholder Clusters}

In the previous chapter, we outlined a stakeholder map (Figure 2.), which is specifically tailored towards public blockchains. The allocation of identified aspects in that model to stakeholders is covered in this chapter, 
based on the analysis of our survey results $(n=74)$. In doing so, we identified the three main stakeholder clusters user-investor (41 out of $n=74)$, user-validator ( 20 out of $\mathrm{n}=74$ ), and validator-investor (18 out of $\mathrm{n}=74$ ), which we will characterize in the following. A stakeholder is assigned to such a cluster if both associated actor types are embodied simultaneously (overlaps are possible). This approach leverages an analysis of drivers and interests in public and permissionless blockchains based entities with different contribution-profiles. The stakeholder clusters above describe the numerically most found constellations of different actor types in our dataset. The profiling of these stakeholder clusters is based on quantitative data, whose analysis we complied in two tables. Both list the respective results for every cluster in a separate column. Table 1. Driver distribution below shows the proportional occurrence of drivers, followed by the weighted importance ratings of design features that are comprised in Table 2. Interest distribution.

user-investor. Behind this cluster are individuals who both use and invest in public blockchain projects. Profit drives $88 \%$ of these stakeholders to partake in such projects, followed by having an alternative to banks, curiosity (both $71 \%$ ), independence (68\%), and knowledge growth (66\%). Altruism (27\%), communication (27\%), and own reputation (7\%) are the least indicated motives in this cluster. The user-investor cluster prioritizes data security \& integrity (4.82), scalability (4.41), and accessibility (4.30) as the top three rated design features. On the lower end of this rating are anonymity (3.58), data standardization (3.45), and regulatory status (3.38).

user-validator. This cluster comprises individuals who both use public blockchain projects and validate transactions on implemented protocols of such projects. Stakeholders assigned to this cluster are $90 \%$ driven by profit, followed by independence $(80 \%)$, curiosity , income (both 75\%), ideology and knowledge growth (both $70 \%$ ). The least indicated drivers were altruism $(30 \%)$, fear of missing out $(20 \%)$, and own reputation $(5 \%)$. Data security \& integrity (4.85), on-chain decentralized governance (4.45), and scalability (4.35) are the top three prioritized design features for this stakeholder cluster, whereas automated conflict resolution (3.58), data standardization (3.45), and regulatory status (2.80) are perceived as least important.

validator-investor. Individuals who both validate transactions on implemented protocols of public blockchain projects and invest in such projects are assigned to this cluster. As in the previous two clusters, profit (94\%) is the most indicated driver. Further, curiosity , income (both 78\%), general mistrust, ideology, independence, and knowledge growth (all $67 \%$ ) profile the validator-investor cluster. As in the previous cluster, the top three rated design features are data security \& integrity (4.83), on-chain decentralized governance (4.33), and scalability (4.28). Anonymity (3.61), data standardization (3.50), regulatory status (2.83) are the lowest ranking aspects. As with the uservalidator cluster, altruism (28\%), fear of missing out $(22 \%)$, and own reputation (6\%) are the drivers with the lowest proportional occurrence.

Table 1. Driver distribution

\begin{tabular}{|c|c|c|c|}
\hline \multirow{2}{*}{$\begin{array}{l}\text { driver (answer } \\
\text { choices) }\end{array}$} & \multicolumn{3}{|c|}{ proportional occurrence } \\
\hline & $\begin{array}{c}\text { user- } \\
\text { investor }\end{array}$ & $\begin{array}{c}\text { user- } \\
\text { validator }\end{array}$ & $\begin{array}{l}\text { validator- } \\
\text { investor }\end{array}$ \\
\hline $\begin{array}{c}\text { access / } \\
\text { affordability }\end{array}$ & $54 \%$ & $55 \%$ & $61 \%$ \\
\hline alternative to bank & $71 \%$ & $60 \%$ & $61 \%$ \\
\hline altruism & $27 \%$ & $30 \%$ & $28 \%$ \\
\hline anonymity & $54 \%$ & $65 \%$ & $61 \%$ \\
\hline $\begin{array}{l}\text { avoid regulation / } \\
\text { censorship }\end{array}$ & $46 \%$ & $45 \%$ & $56 \%$ \\
\hline communication & $27 \%$ & $35 \%$ & $39 \%$ \\
\hline curiosity & $71 \%$ & $75 \%$ & $78 \%$ \\
\hline fear of missing out & $37 \%$ & $20 \%$ & $22 \%$ \\
\hline general mistrust & $56 \%$ & $60 \%$ & $67 \%$ \\
\hline ideology & $63 \%$ & $70 \%$ & $67 \%$ \\
\hline income & $46 \%$ & $75 \%$ & $78 \%$ \\
\hline independence & $68 \%$ & $80 \%$ & $67 \%$ \\
\hline knowledge growth & $66 \%$ & $70 \%$ & $67 \%$ \\
\hline $\begin{array}{c}\text { novel store of } \\
\text { value }\end{array}$ & $51 \%$ & $55 \%$ & $50 \%$ \\
\hline own reputation & $7 \%$ & $5 \%$ & $6 \%$ \\
\hline profit & $88 \%$ & $90 \%$ & $94 \%$ \\
\hline
\end{tabular}

Table 2. Interest distribution

\begin{tabular}{|c|c|c|c|}
\hline \multirow{2}{*}{$\begin{array}{l}\text { design feature } \\
\text { (answer choices) }\end{array}$} & \multicolumn{3}{|c|}{ weighted importance } \\
\hline & $\begin{array}{c}\text { user- } \\
\text { investor }\end{array}$ & $\begin{array}{c}\text { user- } \\
\text { validator }\end{array}$ & $\begin{array}{l}\text { validator- } \\
\text { investor }\end{array}$ \\
\hline accessibility & 4.30 & 3.95 & 4.11 \\
\hline agility & 3.77 & 3.60 & 3.72 \\
\hline anonymity & 3.58 & 3.85 & 3.61 \\
\hline approval speed & 4.08 & 3.85 & 4.06 \\
\hline $\begin{array}{l}\text { automated conflict } \\
\text { resolution }\end{array}$ & 3.69 & 3.58 & 3.89 \\
\hline $\begin{array}{l}\text { data security \& } \\
\text { integrity }\end{array}$ & 4.82 & 4.85 & 4.83 \\
\hline $\begin{array}{c}\text { data } \\
\text { standardization }\end{array}$ & 3.45 & 3.45 & 3.50 \\
\hline $\begin{array}{l}\text { decentralized } \\
\text { governance } \\
\text { (off-chain) }\end{array}$ & 4.00 & 4.05 & 3.89 \\
\hline $\begin{array}{l}\text { decentralized } \\
\text { governance } \\
\text { (on-chain) }\end{array}$ & 4.16 & 4.45 & 4.33 \\
\hline scalability & 4.41 & 4.35 & 4.28 \\
\hline regulatory status & 3.38 & 2.80 & 2.83 \\
\hline
\end{tabular}




\section{Discussion}

Section 4 showed our developed stakeholder map and exemplary survey results from its operationalization. To relate our findings to literature on blockchain stakeholders and blockchain governance, a wider discussion follows. We elaborate our key findings in the paragraphs Context and Stakeholders of Public Blockchains, Dual Roles of Stakeholders, and Shared Intentions, Shared Consensus?, and Generalizing Blockchain Stakeholders.

Context and Stakeholders of Public Blockchains. Stakeholder analysis is a powerful tool in the corporate domain to better understand and, consequently, align project stakeholders so that a common goal can be achieved (i.e., [19]). As such, stakeholder analysis tools help us to identify a given context to which appropriate governance measures, such as structures or mechanisms, can be developed (i.e., the COBIT framework for IT governance [20]). As we argue in our related work, blockchains combine both the mutual dependency of actors to maintain and run the system while, at the same time, avoiding centralized governors to, instead, rely on encoded incentives or coordination processes. This renders stakeholder analysis tools from the corporate domain inappropriate [21]. If a central governor is missing, it would be reasonable to assume that central stakeholders in blockchain systems are understood in depth. But, while first steps have been made in terms of actor [12] or role specification (i.e., [22]), the blockchain governance literature seems to be lacking an in-depth understanding on stakeholders of public blockchains. Consequently, the context against which governance measures are supposed to be developed, remains unclear. This can also be seen on various large so-called decentralized autonomous organizations (DAOs), which are instances of public blockchain systems. Several of these DAOs received significant funding (over several hundred million of USD in several cases [21]) to build their technology and governance structures, but, without live systems and users in practice, stakeholder interests remain blurred. Against this background, our research has shown that studying these stakeholders in-depth can yield an interesting picture: studying actor roles, their drivers (what is the root of the stakeholder's intentions?), and interests in design features (concrete manifestation options that can be weighed off) enables a more realistic stakeholder analysis, and, consequently, allows for better stakeholder management.

Dual Roles of Stakeholders. Our findings have also shown that stakeholders in public blockchain systems seem to inhere various roles at the same time, which extends current research [12]. Indeed, most survey respondents saw themselves as fulfilling at least two types of roles, which led to three stakeholder clusters within this research. Interestingly, all respondents within these clusters indicated to be primarily driven by profit. It can be assumed, that such stakeholders might be inclined to withdraw their capital, if a more profitable opportunity arises. This is significant, because invested capital in blockchain projects determines a token's value, which, in turn, serves as a compensation for blockchain validators. If a token value decreases, miners might step away that, eventually, may render a blockchain system less secure due to lower hash rates or a higher degree of centralization (e.g., [23]). To add to this line of thought, our results also show that the userinvestor cluster in particular is characterized by the fear of missing out on current trends. As a third exemplary finding, our results indicate that the user-validator cluster is primarily driven by the thought of being independent, which is also line with their, in comparison to the other identified clusters, higher rating of ideology. Overall, this may indicate a widening gap between differently contributing stakeholders. Their commitment, and therefore the willingness to contribute resources, may strongly differ in uncertain but decisive times, like sudden price movements or regulation.

Shared Intentions, Shared Consensus? For another, in this research, we were introducing blockchain design features, i.e., interests of public blockchain stakeholders regarding blockchain technology, and related these to our derived stakeholder clusters. In our related work, we introduced the paradox of openness [10], which aims at enabling a maximum openness for mutual innovation, while ensuring value and interest safeguarding for all involved stakeholders. Against this background, we see a need for a coherent, preliminary identification of interests, attributed to distinctive stakeholder clusters. However, the question remains, if there is more between shared expectations/intentions/design features, i.e., alleged shared consensus, and the realization of such visions? For example, are projects failing because of the forces of the BC Environment in stakeholders, i.e., not between the forces within the BC Governance frame? Or perhaps different stakeholders still imagine something different under a concept that is considered equally important, such as data security and integrity, or scalability? Our insights indicate that stakeholder groups have distinct interests. Data security and integrity is the highest rated BC design feature across all stakeholder clusters. Their subsequent prioritization, however, differ. For instance, scalability and accessibility are among the three core interests of the user-investor cluster. Stakeholders who we assigned to a cluster with a validator component, do, however, perceive decentralized on-chain governance as second most important aspect, followed by scalability. 
Consequently, our results indicate that stakeholders appreciate the possibility of raising their voice via votes (common via, for example, smart contracts or systems utilizing Proof-of-Stake). However, their participation in ongoing developments should be channeled to where their actual interest lies, which would require an improved communication concept among stakeholders.

Generalizing Blockchain Stakeholders. Lastly, comparing our identified stakeholder clusters to the ones of related work (such as the value-based stakeholder groups from Wingreen et al. [16]), we conclude that our newly developed framework allows for a more precise representation. We conclude that such clusters should be defined according to embodied actor types (or roles, such as validator), as we did in this research, instead of collating value expectations and a priori label such groups as stakeholder clusters. To counteract the generalization and oversimplification of stakeholder communities in academia, we see this approach as crucial, namely the consideration of contributing roles (actor types) of individuals in the blockchain infrastructure (seen as a platform economy) during the process of stakeholder analysis. We see our framework as a contribution in form of an applicable tool to evaluate primary interests of stakeholders (alongside their rationale behind the prioritization) in public and permissionless blockchains. Consequently, in the overarching process of analyzing the alignment of deviating incentives and interests, our framework could be used as a very first instance to capture these aspects and assign them to stakeholder clusters (which are defined by their contribution to the project) in a coherent and uniform approach.

We are aware that different application areas of (public) blockchain systems, as for instance described by Schlegel et al. [15], might infer different prioritizations of aspects per use case. However, our framework captures the aspects that are very essential and characteristic for pubic blockchains in the first place. Accordingly, the evaluated interests per stakeholder cluster must be understood as such, i.e., not as a manifestation and outline of a specific blockchain application. We argue that this approach should enable a general classification of public blockchains in the context of decentralized collaboration, and, hence, point out the primary interests of different stakeholder clusters, as they fundamentally shape such projects. The assimilation of these insights might enable a further assessment of blockchains in terms of its eventual capacity to allow for decentralized collaboration.

\section{Conclusion}

In the context of blockchain governance, we developed a framework (stakeholder map) that captures the interests (reflected in $B C$ Design Features), as well as motives and incentives (Drivers) of stakeholders in public and permissionless blockchain projects. We operationalized this framework and conducted the resulting survey on several online platforms to gather data from communities of current public blockchains. Our analysis of this data yielded three main stakeholder clusters: user-investor, user-validator, and validatorinvestor. As such, this research provides a novel tool for stakeholder analysis in academia and practice to improve blockchain governance.

Alongside the identification of shared interests, such as data security and integrity, we found that every stakeholder cluster can be distinctively characterized in terms of what drives these individuals to participate in public blockchains in the first place. This highlights that differently contributing stakeholders, even though they might show mutual interests, are not homogenously embedded in our outlined stakeholder map. This indicates a possible widening gap between such individuals, for example, regarding their level of commitment to the projects they are involved in. To utilize blockchain technology by the means of collaboration in a decentralized manner, these insights must be considered.

From a methodological standpoint, this research must admit several limitations. Since we did not have the (technical) possibility to backtrack the survey responses to their publication source, the results might be skewed towards a rather distinctive community, e.g., if most of the survey responses originated form a single online platform dedicated to a single, rather unique (and thus not representative) public blockchain project. We were aware of this issue and strived for an adequately broad distribution of the survey. Furthermore, we were not able to analyze the (primary) interests of certain actors in our framework, as there were not enough responses in this regard to conduct a sophisticated data analysis. Even though we strove to justify our applied methods, we are aware that, for example, the applied equations and methods in our conducted data analysis form only a small fraction of all possible approaches.

Based on our research, we see several possible future research opportunities. For one, a further classification of our identified drivers based on psychological heuristics could reveal additional insights regarding the behavioral and motivational rationale of stakeholders in public blockchains. An example would be the examination of the overjustification effect, by differentiating between intrinsic and extrinsic drivers. For another, one could focus on the dynamics of our outlined stakeholder map. For example, which criteria are crucial, which interests must be met or undercut that a certain stakeholder participates or abandons a blockchain project? Lastly, our developed framework 
could form the basis for an even more sophisticated approach to capture the interests of stakeholders in public and permissionless blockchain projects.

\section{References}

[1] Nakamoto, S. (2008). Bitcoin: A Peer-to-Peer Electronic Cash System [White Paper]. Retrieved July 30, 2020, from https://bitcoin.org/bitcoin.pdf

[2] De Filippi, P., and Loveluck, B. (2016). The invisible politics of Bitcoin: governance crisis of a decentralised infrastructure. Internet Policy Review, Alexander von Humboldt Institute for Internet and Society. hal01382007

[3] de Laat, P. B. (2007). Governance of open source software: state of the art. Journal of Management \& Governance, 11(2), 165-177. doi:10.1007/s10997-007-9022-9

[4] DiRose, S., and Mansouri, M. (2018, June). Comparison and analysis of governance mechanisms employed by blockchain-based distributed autonomous organizations. In 2018 13th Annual Conference on System of Systems Engineering (SoSE) (pp. 195-202). IEEE. doi:10.1109/SYSOSE.2018.8428782

[5] Azouvi S., Maller M., and Meiklejohn S. (2019). Egalitarian Society or Benevolent Dictatorship: The State of Cryptocurrency Governance. In: Zohar A. et al. (eds) Financial Cryptography and Data Security. FC 2018. Lecture Notes in Computer Science, vol 10958. Springer, Berlin, Heidelberg. doi:10.1007/978-3-66258820-8 10

[6] Hsieh, Y., (JP) Vergne, J., and Wang, S. (2017). The internal and external governance of blockchain-based organizations: Evidence from cryptocurrencies. In Bitcoin and Beyond (1st ed., Vol. 1, pp. 48-68). Routledge. doi:10.4324/9781315211909-3

[7] Briggs, K. (2018). Taming the Wild West: How the SEC Can Legitimize Initial Coin Offerings (ICOs), Protect Consumers from Bad Actors, and Encourage Blockchain Development. Bus. Entrepreneurship \& Tax L. Rev., 2, 424. Retrieved June 15, 2021, from https://scholarship.law.missouri.edu/betr/vol2/iss $2 / 9$

[8] Weill, P. (2004). Don't just lead, govern: How topperforming firms govern IT. MIS Quarterly Executive, 3(1), 1-17. Retrieved April 16, 2021, from http://www.gti4u.es/curso/material/complementario/we ill 2004.pdf

[9] Beck, R., Müller-Bloch, C., and King, J. L. (2018). Governance in the blockchain economy: A framework and research agenda. Journal of the Association for Information Systems, 19(10), 1. doi: $10.17705 / 1$ jais.00518

[10] Schmeiss, J., Hoelzle, K., and Tech, R. P. G. (2019). Designing Governance Mechanisms in Platform Ecosystems: Addressing the Paradox of Openness through Blockchain Technology. California Management Review, 62(1), 121-143. doi: $10.1177 / 0008125619883618$

[11] Lumineau, F., Wang, W., and Schilke, O. (2020). Blockchain Governance-A New Way of Organizing
Collaborations? Organization Science. doi: 10.1287/orsc. 2020.1379

[12] Islam, N., Mäntymäki, M., and Turunen, M. (2019, January). Understanding the role of actor heterogeneity in blockchain splits: an actor-network perspective of bitcoin forks. In Proceedings of the 52nd Hawaii International Conference on System Sciences. doi: $10.24251 /$ HICSS.2019.556

[13] Rossi, M., Mueller-Bloch, C., Thatcher, J. B., and Beck, R. (2019). Blockchain research in information systems: Current trends and an inclusive future research agenda. Journal of the Association for Information Systems, 20(9), 14. doi:10.17705/1 jais.00571

[14] Musiani, F., Mallard, A., and Méadel, C. (2017). Governing what wasn't meant to be governed: A controversy-based approach to the study of Bitcoin governance. In Bitcoin and Beyond: Cryptocurrencies, Blockchains, and Global Governance (pp. 133-156). Taylor and Francis. doi:10.4324/9781315211909-7

[15] Schlegel, M., Zavolokina, L., and Schwabe, G. (2018, January). Blockchain Technologies from the Consumers' Perspective: What Is There and Why Should Who Care?. In Proceedings of the 51st Hawaii International Conference on System Sciences. doi:10.24251/HICSS.2018.441

[16] Wingreen, S., Kavanagh, D., John Ennis, P., and Miscione, G. (2020). Sources of Cryptocurrency Value Systems: The Case of Bitcoin. International Journal of Electronic Commerce, 24(4), 474-496. doi:10.1080/10864415.2020.1806469

[17] Eyal, I., and Sirer, E. G. (2014, March). Majority is not enough: Bitcoin mining is vulnerable. In International Conference on Financial Cryptography and Data Security (pp. 436-454). Springer, Berlin, Heidelberg. doi: $10.1145 / 3212998$

[18] Ketsdever, S., and Fischer, M. J. (2019, October). Incentives Don't Solve Blockchain's Problems. In 2019 IEEE 10th Annual Ubiquitous Computing, Electronics \& Mobile Communication Conference (UEMCON) ( $\mathrm{pp}$. 0873-0876).

IEEE. doi:10.1109/UEMCON47517.2019.8992984

[19] Kates, A., and Galbraith, J. R. (2010). Designing your organization: Using the STAR model to solve 5 critical design challenges. John Wiley \& Sons.

[20] ISACA (2021). COBIT. Retrieved June 15, 2021, from https://www.isaca.org/resources/cobit

[21] Ziolkowski, R., Miscione, G., and Schwabe, G. (2020). Exploring Decentralized Autonomous Organizations: Towards Shared Interests and 'Code is Constitution'. doi:10.5167/uzh-193663

[22] Peters, G. W., and Panayi, E. (2016). Understanding modern banking ledgers through blockchain technologies: Future of transaction processing and smart contracts on the internet of money. In Banking beyond banks and money (pp. 239-278). Springer, Cham.

[23] Kano, Y., and Nakajima, T. (2018). A novel approach to solve a mining work centralization problem in blockchain technologies. International Journal of Pervasive Computing and Communications, 14(1), 1532. doi:10.1108/IJPCC-D-18-00005 